\title{
Pulsatile Release Intraruminal Device
}

National Cancer Institute

\section{Source}

National Cancer Institute. Pulsatile Release Intraruminal Device. NCI Thesaurus. Code C149879.

Solid sing le-dose preparation intended for oral use in ruminant animals. It is designed to be retained in the rumen to deliver the active substance(s) in a pulsatile manner by the release of a specific quantity of active substance(s) at one or several defined intermediate intervals. This may be achieved by corrosion by ruminal fluids of the metallic elements of the intraruminal device which leads to sequential release of the constituent units which are usually in the form of tablets. 